\title{
Research of the Microscopic Polarizability Tensors for the Third and Fourth-Order Nonlinear Spectroscopy Yuan WANG ${ }^{1, \text { a }}$, Xueyong DING ${ }^{1}$, Liansheng WANG ${ }^{1}$ \\ ${ }^{1}$ Department Of Polytechnic, Sanya University, Sanya, 572022,China \\ aemail: wangyuan0155@163.com
}

Keywords: Coherent Anti-Stokes Raman Spectra; Coherent Anti-Stokes Hyper-Raman Spectra; Microscopic Polarizability Tensors

\begin{abstract}
Knowledge of the ratios between different polarizability tensor elements of a chemical group in a molecule is crucial for quantitative interpretation and polarization analysis of its coherent anti-stokes raman spectra (CARS) and coherent anti-stokes hyper-raman spectra (CAHRS). The hyperpolarizability derivative model along with experimentally obtained Raman and Hyper-Raman depolarization ratios could be used to obtain such tensor ratios for the $\mathrm{CH}$, $\mathrm{CH} 2$, and $\mathrm{CH}$ groups. Such treatment can be used to quantitatively analyze and simulate the intensity and field polarization dependence in the CARS and CAHRS spectra for the symmetric and asymmetric stretching modes of $\mathrm{CH} 3$ groups, respectively.
\end{abstract}

\section{Introduction}

Higher-order nonlinear spectroscopic techniques, such as the third order Coherent Anti-Stokes Raman Spectroscopy(CARS) and the fourth order Coherent Anti-Stokes Hyper-Raman Spectroscopy (CAHRS) have been recently employed to investigate or imaging molecular interfaces and biological membranes[1-10]. Being Raman spectroscopy, the clear advantage of the CARS and the CAHRS is that they do not directly use the infrared laser pulses that generally have trouble to penetrate the condensed phase as in the ordinary IR or SFG vibrational spectroscopy, for obtaining the coherent molecular vibrational spectra of the buried interfaces and membranes. The fourth order CAHRS process is particularly interesting because it is also an interface specific process just as the SFG-VS. In addition, it is worth noting that Under the electric dipole approximation the CARS haven't surface selectivity, but it could study the films, and get the information about the structure and component of the films and so on.[11-13] But in the past two decades, the high order nonlinear spectroscopy are used to qualitative studied the molecules in the interface, and the quantitative study is to very hard.

In this article[14], we had discussed the various connection of the macroscopic susceptibility and the microscopic polarizability tensors of the third and fourth-order nonlinear spectroscopy of ordered molecular system, and calculate the expressions of them. But toward the CARS and the CAHRS, the number of independent elements of molecular microcosmic nonlinear hyperpolarizability $\beta_{i^{\prime} j^{\prime} k^{\prime} l}$ ' and $\beta_{i^{\prime} j^{\prime} k^{\prime} l^{\prime} m}$ ' are much toward the $C_{3 \mathrm{~V}}$ symmetry molecular.

\section{Simplify the molecular microcosmic nonlinear hyperpolarizability of the CARS and CAHRS}

Spectroscopy measures the interactions between electromagnetic fields and molecular system. Through spectroscopy, we are able to obtain information on molecular structure, conformation and dynamics. Naturally, the way in the application of spectroscopy lies in the way to obtain microscopic or molecular information from the macroscopic spectroscopic measurement.

The key for such quantitative interpretation lies on the ability to obtain or estimate the ratios between different microscopic polarizability $\beta_{i^{\prime} j^{\prime} k^{\prime} l}$ ' tensor elements of a chemical group in a molecule. So far, almost all of the efforts have focused on the stretching vibrational modes. Toward the CARS, fix one of the incident light's frequency $\omega_{1}$, and scan the other incident light's frequency $\omega_{2}$, when the difference of $\omega_{1}$ and $\omega_{2}$ equal to the inherent vibration frequency of the studied 
molecular, the signal would be resonant enhanced and could get the corresponding vibration spectroscopy of the study molecular. The expression of the vibrationally resonant part of $\beta_{i{ }^{\prime} j}{ }^{\prime} k^{\prime} l^{\prime}$, is derived from the electric dipole approximation for the interaction of molecules and the light waves as follows[15]:

$$
\beta_{i j^{\prime} k^{\prime \prime}}=\sum_{v} \frac{\beta_{i j^{\prime} k^{\prime \prime} l^{\prime}}^{q, R}}{\omega_{1}-\omega_{2}-\omega_{v g}+i \Gamma_{v g}}\left(\rho_{g}-\rho_{\nu}\right)+\beta_{i j^{\prime} k^{\prime} l^{\prime}}^{q, N R}
$$

in which, the vibrationally resonant term $\beta_{i j^{\prime} k^{\prime \prime}}^{q, R}$ show as:

$$
\begin{aligned}
& \beta_{i j^{\prime} k^{\prime \prime}}^{q, R}=-\frac{1}{\hbar} \times \sum_{n^{\prime \prime}} \frac{1}{\hbar}\left(\frac{\left\langle g\left|e \times r\left(\omega_{C A R S}\right)_{i^{\prime}}\right| n^{\prime \prime}\right\rangle\left\langle n^{\prime \prime}\left|e \times r\left(\omega_{1}\right)_{j^{\prime}}\right| v\right\rangle}{\omega_{C A R S}-\omega_{n^{\prime \prime} g}}-\frac{\left\langle g\left|e \times r\left(\omega_{1}\right)_{j^{\prime}}\right| n^{\prime}\right\rangle\left\langle n^{\prime}\left|e \times r\left(\omega_{C A R S}\right)_{i^{\prime}}\right| v\right\rangle}{\omega_{1}+\omega_{n^{\prime \prime} g}}\right) \\
& \times \sum_{n^{\prime}} \frac{1}{\hbar}\left(\frac{\left\langle v\left|e \times r\left(\omega_{1}\right)_{k^{\prime}}\right| n^{\prime}\right\rangle\left\langle n^{\prime}\left|e \times r\left(\omega_{2}\right)_{I^{\prime}}\right| g\right\rangle}{\omega_{1}-\omega_{n^{\prime} g}}-\frac{\left\langle v\left|e \times r\left(\omega_{2}\right)_{I^{\prime}}\right| n^{\prime}\right\rangle\left\langle n^{\prime}\left|e \times r\left(\omega_{1}\right)_{k^{\prime}}\right| g\right\rangle}{\omega_{2}+\omega_{n^{\prime} g}}\right) \\
& {\left[\begin{array}{l}
\left.-n^{\prime \prime}\right\rangle \\
\left.-n^{\prime}\right\rangle
\end{array}\right.}
\end{aligned}
$$
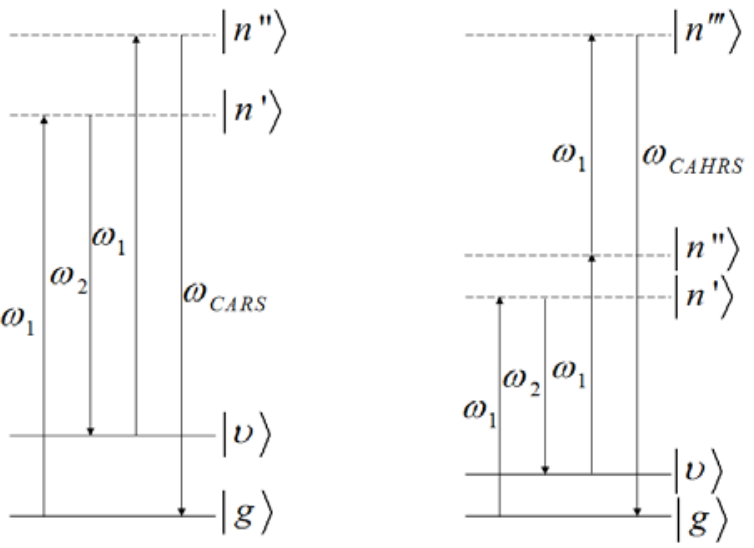

(a) (b)

Fig.1. (a): schematic transitions for coherent anti-stokes raman spectroscopy; (b): schematic transitions for coherent anti-stokes hyper-raman spectroscopy. $|g\rangle$ and $|v\rangle$ represent the ground state(real) and the vibration excited states(real), $\left|n^{\prime}\right\rangle,\left|n^{\prime \prime}\right\rangle$ and $\left|n^{\prime \prime \prime}\right\rangle$ represent the arbitrary excited states(virtual or real), respectively.

in which, $i$ ', $j$ ', $k$ 'and l'represent the polarization of the radiation of frequency $\omega_{\text {CARs }}, \omega_{1}$ (transition from $|v\rangle$ state to $\left|n^{\prime \prime}\right\rangle$ state in the Fig.1), $\omega_{1}$ (transition from $|v\rangle$ state to $\left|n^{\prime}\right\rangle$ state in the Fig.1) and $\omega_{2}$, respectively; $\omega_{v g}$ and $\Gamma_{v g}$ are the frequency and the damping width of the vibrational transition from the electronic ground state $|g\rangle$ to upper vibrational level $|v\rangle$, respectively; $\rho_{g}$ and $\rho_{v}$ are the fractional population of $|g\rangle$ and $|v\rangle$ levels, respectively. It is seen from Eq.(2) that $\beta_{i j^{\prime} k^{\prime} l^{\prime}}^{q, R}$ is the product of the $\left(k^{\prime} l^{\prime}\right)$ component of the Stokes Raman tensor $\partial \alpha_{k^{\prime} l^{\prime}}^{s} / \partial Q_{q}$ for the $g \rightarrow v$ transition and the $\left(i^{\prime} j^{\prime}\right)$ component of the Anti-Stokes Raman tensor $\partial \alpha_{i j^{\prime}}^{a} / \partial Q_{q}$ for the $v \rightarrow g$ transition[15-17]:

$$
\beta_{i j^{\prime} k^{\prime} \prime^{\prime}}^{q, R}=-\frac{1}{2 \omega_{q} \varepsilon_{0}} \frac{\partial \alpha_{i j^{\prime}}^{a}}{\partial Q_{q}} \frac{\partial \alpha_{k^{\prime \prime} l^{\prime}}^{s}}{\partial Q_{q}} \approx-\frac{1}{2 \omega_{q} \varepsilon_{0}}\left(\frac{\partial \alpha_{i j^{\prime}}^{s}}{\partial Q_{q}}\right)^{*} \frac{\partial \alpha_{k^{\prime} l^{\prime}}^{s}}{\partial Q_{q}}
$$

in which,

$$
\begin{aligned}
& \frac{\partial \alpha_{i j^{\prime}}^{a}}{\partial Q_{q}}=\sqrt{\frac{2 \omega_{q} \varepsilon_{0}}{\hbar}} \sum_{n^{\prime \prime}} \frac{1}{\hbar}\left(\frac{\left\langle g\left|e \times r\left(\omega_{C A R S}\right)_{i^{\prime}}\right| n^{\prime \prime}\right\rangle\left\langle n^{\prime \prime}\left|e \times r\left(\omega_{1}\right)_{j^{\prime}}\right| v\right\rangle}{\omega_{C A R S}-\omega_{n^{\prime \prime} g}}-\frac{\left\langle g\left|e \times r\left(\omega_{1}\right)_{j^{\prime}}\right| n^{\prime \prime}\right\rangle\left\langle n^{\prime \prime}\left|e \times r\left(\omega_{C A R S}\right)_{i^{\prime}}\right| v\right\rangle}{\omega_{1}+\omega_{n^{\prime \prime} g}}\right) \\
& \frac{\partial \alpha_{k^{\prime} \prime^{\prime}}^{s}}{\partial Q_{q}}=\sqrt{\frac{2 \omega_{q} \varepsilon_{0}}{\hbar}} \sum_{n^{\prime}} \frac{1}{\hbar}\left(\frac{\left\langle v\left|e \times r\left(\omega_{1}\right)_{k^{\prime}}\right| n^{\prime}\right\rangle\left\langle n^{\prime}\left|e \times r\left(\omega_{2}\right)_{I^{\prime}}\right| g\right\rangle}{\omega_{1}-\omega_{n^{\prime} g}}-\frac{\left\langle v\left|e \times r\left(\omega_{2}\right)_{l^{\prime}}\right| n^{\prime}\right\rangle\left\langle n^{\prime}\left|e \times r\left(\omega_{1}\right)_{k^{\prime}}\right| g\right\rangle}{\omega_{2}+\omega_{n^{\prime} g}}\right)
\end{aligned}
$$


here we have included the factor of $\varepsilon_{0}$ in the Eq.(3) because it is a convention issue.

The intensity of CARS signal of $q$ th vibration mode is proportional to the square of $\beta_{i j^{\prime} k^{\prime} \prime^{\prime}}^{q, R}$, and Eq.(3) shows that we can estimate the relative magnitudes among vibration modes from the intensities of stokes and anti-stokes Raman spectra, and the superscripts 's' and 'a' denote the stokes and anti-stokes. we should note that we have omit the imaginary part of the denominator in the Eq.(4) and Eq.(5), so we could get that $\alpha_{i j^{\prime}}^{a}=\left(\alpha_{i j^{\prime}}^{s}\right)^{*}$ in Eq.(3), where $\left(\alpha_{i j^{\prime}}^{s}\right)^{*}$ is the conjugate complex of $\alpha_{i j^{\prime}}^{\mathrm{s}}$. The Fig.(1-a) show the schematic transitions of the coherent anti-stokes raman spectroscopy, from which we also could get the conclusion that $\beta_{i j^{\prime} k^{\prime} l^{\prime}}^{q, R}$ is the product of the $\left(k^{\prime} l^{\prime}\right)$ component of the Stokes Raman tensor $\partial \alpha_{k^{\prime} \prime^{\prime}}^{s} / \partial Q_{q}$ and the $\left(i j^{\prime}\right)$ component of the Anti-Stokes Raman tensor $\partial \alpha_{i j^{\prime}}^{a} / \partial Q_{q}$.

From the above discuss, we could get the approaches employed to obtain the $\beta_{i{ }^{\prime} j^{\prime}{ }^{\prime} \text { 'l }}$, tensor ratios are all based on the following general relationship between the third order hyperpolarizability tensors $\left(\beta_{i j^{\prime} k^{\prime} \prime^{\prime}}^{q, R}\right)$ for a particular vibrational mode $q$ to the anti-stokes Raman polarizability derivative tensors $\left(\partial \alpha_{i j^{\prime}}^{a} / \partial Q_{q}\right.$, usually denoted as $\left.\alpha_{i j^{\prime}}^{\prime a}\right)$ and the stokes Raman polarizability derivative tensors $\left(\partial \alpha_{k^{\prime}}^{s} / \partial Q_{q}\right.$, usually denoted as $\alpha_{k^{\prime} l^{\prime}}^{\prime s}$ ) of the same ( $q$ th) mode. Therefore, the Eq.(3) could be express as:

$$
\beta_{i j^{\prime} k^{\prime \prime} \prime^{\prime}}^{q, R}=-\frac{1}{2 \omega_{q} \varepsilon_{0}}\left(\alpha_{i j^{\prime}}^{\prime s}\right)^{*} \alpha_{k^{\prime \prime} \prime^{\prime}}^{\prime s}
$$

Therefore, according to Eq.(6), if the proper ratios between different $\alpha_{i j^{\prime}}^{\prime a}$ and $\alpha_{k^{\prime \prime}}^{\prime s}$ terms are known, the ratios of $\beta_{i j^{\prime} k^{\prime \prime}}^{q, R}$ elements can be readily obtained.

At the same time, we should note that the stokes and anti-stokes Raman tensor elements in the Eq.(3) have the same transition frequency from the Fig.(1-a) and the Eq.(4) and Eq.(5), so them must correspond to the same molecular vibrational mode, symmetric or antisymmetric. If the connections of the Raman tensor elements of the symmetric and antisymmetric mode have be known, we could more simplify the connection of the CARS tensor elements of the symmetric and antisymmetric mode, respectively.

And follow the Eq.(1) and Eq.(3), The microscopic polarizability $\beta_{i^{\prime} j^{\prime} k^{\prime} l^{\prime} m}$ ' could show that $[16,18]$

$$
\beta_{i j^{\prime} k^{\prime} I^{\prime} m^{\prime}}=\sum_{v} \frac{\beta_{i j^{\prime} k^{\prime} \prime^{\prime} m^{\prime}}^{q, R}}{\omega_{1}-\omega_{2}-\omega_{v g}+i \Gamma_{v g}}\left(\rho_{g}-\rho_{v}\right)+\beta_{i j^{\prime} k^{\prime} I^{\prime} m^{\prime}}^{q, N R}
$$

in which, the vibrationally resonant term $\beta_{i j^{\prime} k^{\prime \prime} \prime m^{\prime}}^{q, R}$ show as:

$$
\beta_{i^{\prime} j^{\prime} k^{\prime \prime} m^{\prime} m^{\prime}}^{q, R}=-\frac{1}{2 \omega_{q} \varepsilon_{0}} \frac{\partial \beta_{i j^{\prime} k^{\prime}}^{a}}{\partial Q_{q}} \frac{\partial \alpha_{l^{\prime} m^{\prime}}^{s}}{\partial Q_{q}}=-\frac{1}{2 \omega_{q} \varepsilon_{0}} \beta_{i j^{\prime} k^{\prime}}^{\prime a} \alpha_{l^{\prime} m^{\prime}}^{\prime s}
$$

in which, $\alpha_{I^{\prime} m^{\prime}}^{\prime s}$ is the Stokes Raman tensor and $\beta_{i j^{\prime} k^{\prime}}^{\prime a}$ is the Hyper-Raman tensor.

\section{Simplify the Stokes Raman tensor and Hyper-Raman tensor}

For the stretching vibrational modes of a molecular group with $C_{3 \mathrm{~V}}$ symmetry, show in the Fig.(2-a), there are a single symmetric mode $\left(A_{1}\right)$ and a doubly degenerated asymmetric mode $(E)$. The nonzero Raman tensor elements $\alpha_{i j^{\prime}}^{\prime}$ for the $C_{3 \mathrm{~V}}$ symmetric stretching vibrational modes are as follows:

For the $C_{3 \mathrm{~V}-\mathrm{A} 1}$ symmetric mode:

Raman: $\alpha_{a a}^{\prime S S}=\alpha_{b b}^{\prime S S}\left(=R_{S S}^{R} \alpha_{c c}^{\prime S S}\right), \alpha_{c c}^{\prime s S}$

For the $C_{3 \mathrm{~V}-\mathrm{E}}$ symmetric mode: 
Raman: $\alpha_{a c}^{\prime A S}=\alpha_{c a}^{\prime A S}=\alpha_{b c}^{\prime A S}=\alpha_{c b}^{\prime A S}, \alpha_{a a}^{\prime A S}=-\alpha_{b b}^{\prime A S}=-\alpha_{a b}^{\prime A S}=-\alpha_{b a}^{\prime A S}\left(=R_{A S}^{R} \alpha_{a c}^{\prime A S}\right)$

where, $R_{S S}^{R}$ and $R_{A S}^{R}$ are two $\alpha_{i j^{\prime}}^{\prime}$ elements ratios, which are the real number. And the nonzero Hyper-Raman tensor elements $\beta_{i j^{\prime} k^{\prime}}^{\prime}$ for the $C_{3 \mathrm{~V}}$ symmetric stretching vibrational modes are as follows:

For the $C_{3 \mathrm{~V}-\mathrm{A} 1}$ symmetric mode:

Hyper-Raman: $\beta_{a a a}^{\prime S S}=-\beta_{a b b}^{\prime S S}=-\beta_{b a b}^{\prime S S}=-\beta_{b b a}^{\prime S S}\left(=R_{S S, 1}^{H R} \beta_{c c c}^{\prime S S}\right), \beta_{c a a}^{\prime S S}=\beta_{c b b}^{\prime S S}\left(=R_{S S, 2}^{H R} \beta_{c c c}^{\prime S S}\right)$

$$
\beta_{a a c}^{\prime S S}=\beta_{a c a}^{\prime S S}=\beta_{b b c}^{\prime S S}=\beta_{b c b}^{\prime S S}=\left(R_{S S, 3}^{H R} \beta_{c c c}^{\prime S S}\right), \beta_{c c c}^{\prime S S}
$$

For the $C_{3 \mathrm{~V}-E}$ symmetric mode:

$$
\begin{aligned}
\text { Hyper-Raman: } \beta_{a a b}^{\prime A S} & =\beta_{a b a}^{\prime A S}=\beta_{b b a}^{\prime A S}=\beta_{b a b}^{\prime A S}\left(=R_{A S, 1}^{H R} \beta_{a a a}^{\prime A S}\right), \quad \beta_{a b b}^{\prime A S}=\beta_{b a a}^{\prime A S}\left(=R_{A S, 2}^{H R} \beta_{a a a}^{\prime A S}\right) \\
\beta_{c c a}^{\prime A S} & =\beta_{c a c}^{\prime A S}=\beta_{c c b}^{\prime A S}=\beta_{c b c}^{\prime A S}\left(=R_{A S, 3}^{H R} \beta_{a a a}^{\prime A S}\right), \quad \beta_{a c c}^{\prime A S}=\beta_{b c c}^{\prime A S}\left(=R_{A S, 4}^{H R} \beta_{a a a}^{\prime A S}\right) \\
\beta_{a a c}^{\prime A S} & =\beta_{a c a}^{\prime A S}=\beta_{c a a}^{\prime A S}=-\beta_{b b c}^{\prime A S}=-\beta_{b c b}^{\prime A S}=-\beta_{c b b}^{\prime A S}=-\beta_{a b c}^{\prime A S}=-\beta_{a c b}^{\prime A S}=-\beta_{b a c}^{\prime A S} \\
& =-\beta_{b c a}^{\prime A S}=-\beta_{c a b}^{\prime A S}=-\beta_{c b a}^{\prime A S}\left(=R_{A S, 5}^{H R} \beta_{a a a}^{\prime A S}\right), \quad \beta_{b b b}^{\prime A S}=\beta_{a a a}^{\prime A S}
\end{aligned}
$$

where, $R_{S S, 1}^{H R}, R_{S S, 2}^{H R}, R_{S S, 3}^{H R}, R_{A S, 1}^{H R}, R_{A S, 2}^{H R}, R_{A S, 3}^{H R}, R_{A S, 4}^{H R}$ and $R_{A S, 5}^{H R}$ are two $\beta_{i j^{\prime} k^{\prime}}^{\prime}$ elements ratio, which are the real number.

Using the above methods, could get the Stokes Raman tensor and Hyper-Raman tensor of molecular group with $C_{2 \mathrm{~V}}$ symmetry and the $C_{\infty \mathrm{V}}$ symmetry.

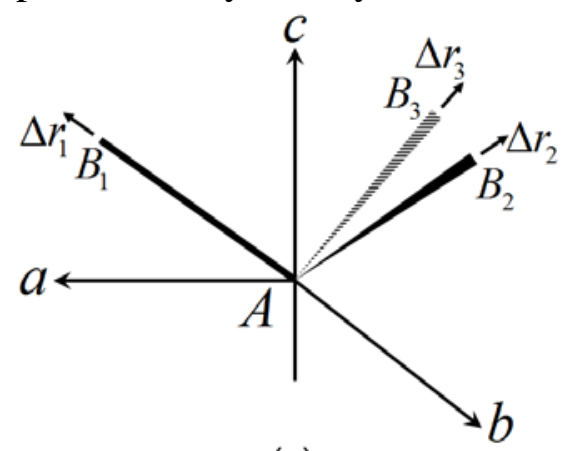

(a)

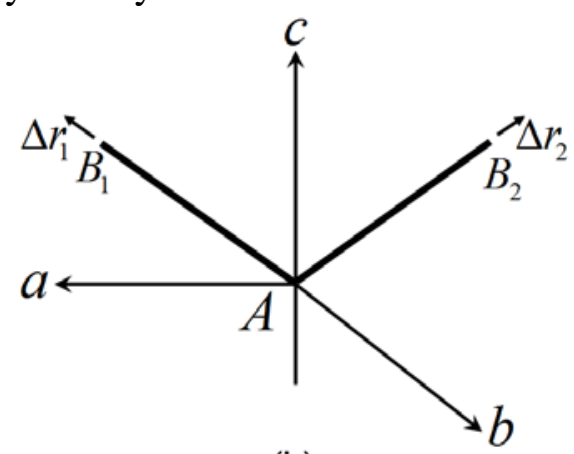

(b)

Fig.2. Molecule-fixed (a,b,c) axis for $C_{3 \mathrm{~V}}$ and $C_{2 \mathrm{~V}}$ symmetry. Illustrated with the $A B_{3}$ and $A B_{2}$ group.

\section{Results}

Using the connection of the Eq.(6) and Eq.(8) to Eq.(12), we could simplify the symmetric and antisymmetric $\beta_{i{ }^{\prime} j^{\prime} k^{\prime} l}$ ' and $\beta_{i^{\prime} j^{\prime} k^{\prime} l^{\prime} m}$ ' tensor elements for the $C_{3 \mathrm{~V}}$ symmetry molecule, and show the nonzero CARS and CAHRS tensor elements for the $C_{3 \mathrm{~V}}$ symmetric stretching vibrational modes are as follows:

For the $C_{3 \mathrm{~V}-\mathrm{A1}}$ symmetric mode:

CARS: $\beta_{\text {aaaa }}^{q, S S}=\beta_{b b b b}^{q, S S}=\beta_{\text {aabb }}^{q, S S}=\beta_{b b a a}^{q, S S}\left(=R_{S S}^{R 2} \beta_{c c c c}^{q, S S}\right)$

$$
\beta_{\text {aacc }}^{q, S S}=\beta_{b b c c}^{q, S S}=\beta_{c c a a}^{q, S S}=\beta_{c c b b}^{q, S S}\left(=R_{S S}^{R} \beta_{c c c c}^{q, S S}\right), \quad \beta_{c c c c}^{q, S S}
$$

CAHRS: $\beta_{c c c a a}^{q, S S}=\beta_{c c c b b}^{q, S S}\left(=R_{s S}^{R} \beta_{c c c c c}^{q, S S}\right), \quad \beta_{c a a c c}^{q, S S}=\beta_{c b b c c}^{q, S S}\left(=R_{s S, 3}^{H R} \beta_{c c c c c}^{q, S S}\right)$

$$
\begin{aligned}
& \beta_{\text {caaaa }}^{q, S S}=\beta_{c a a b b}^{q, S S}=\beta_{c b b a a}^{q, S S}=\beta_{c b b b b}^{q, S S}\left(=R_{S S}^{R} R_{S S, 3}^{H R} \beta_{c c c c c}^{q, S S}\right) \\
& \beta_{\text {aaccc }}^{q, S S}=\beta_{\text {acacc }}^{q, S S}=\beta_{b b c c c}^{q, S S}=\beta_{b c b c c}^{q, S S}\left(=R_{S S, 2}^{H R} \beta_{c c c c c}^{q, S S}\right), \beta_{c c c c c}^{q, S S} \\
& \beta_{\text {aacaa }}^{q, S S}=\beta_{\text {aacbb }}^{q, S S}=\beta_{\text {acaaa }}^{q, S S}=\beta_{\text {acabb }}^{q, S S}=\beta_{b b c a a}^{q, S S}=\beta_{b b c b b}^{q, S S}=\beta_{b c b a a}^{q, S S}=\beta_{b c b b b}^{q, S S}\left(=R_{S S}^{R} R_{S S, 2}^{H R} \beta_{c c c c c}^{q, S S}\right)
\end{aligned}
$$

For the $C_{3 \mathrm{~V}-\mathrm{E}}$ symmetric mode: 


$$
\begin{aligned}
& \text { CARS: } \beta_{a c a c}^{q, A S}=\beta_{a c c a}^{q, A S}=\beta_{c a a c}^{q, A S}=\beta_{c a c a}^{q, A S}=\beta_{b c b c}^{q, A S}=\beta_{b c c b}^{q, A S}=\beta_{c b b c}^{q, A S}=\beta_{c b c b}^{q, A S} \\
& \beta_{\text {aaaa }}^{q, A S}=\beta_{b b b b}^{q, A S}=-\beta_{\text {aabb }}^{q, A S}=-\beta_{b b a a}^{q, A S}=\beta_{a b a b}^{q, A S}=\beta_{a b b a}^{q, A S}=\beta_{b a a b}^{q, A S}=\beta_{b a b a}^{q, A S}\left(=R_{A S}^{R}{ }^{2} \beta_{a c a c}^{q, A S}\right) \\
& \text { CAHRS: } \beta_{\text {aaaac }}^{q, A S}=\beta_{\text {aaaca }}^{q, A S}=\beta_{b b b b c}^{q, A S}=\beta_{b b b c b}^{q, A S}, \quad \beta_{a b b a c}^{q, A S}=\beta_{\text {abbca }}^{q, A S}=\beta_{b a a b c}^{q, A S}=\beta_{b a a c b}^{q, A S}\left(=R_{A S, 2}^{H R} \beta_{\text {aaaac }}^{q, A S}\right) \\
& \beta_{\text {accac }}^{q, A S}=\beta_{\text {accca }}^{q, A S}=\beta_{b c c b c}^{q, A S}=\beta_{b c c c b}^{q, A S}\left(=R_{A S, 4}^{H R} \beta_{\text {aaaac }}^{q, A S}\right) \\
& \beta_{c c a a c}^{q, A S}=\beta_{c c a c a}^{q, A S}=\beta_{c a c a c}^{q, A S}=\beta_{c a c c a}^{q, A S}=\beta_{c c b b c}^{q, A S}=\beta_{c c b c b}^{q, A S}=\beta_{c b c b c}^{q, A S}=\beta_{c b c c b}^{q, A S}\left(=R_{A S, 3}^{H R} \beta_{\text {aaaac }}^{q, A S}\right) \\
& \beta_{\text {aabbc }}^{q, A S}=\beta_{\text {aabcb }}^{q, A S}=\beta_{\text {ababc }}^{q, A S}=\beta_{\text {abacb }}^{q, A S}=\beta_{b b a a c}^{q, A S}=\beta_{b b a c a}^{q, A S}=\beta_{\text {babac }}^{q, A S}=\beta_{\text {babca }}^{q, A S}\left(=R_{A S, 1}^{H R} \beta_{\text {aaaac }}^{q, A S}\right) \\
& \beta_{\text {aacaa }}^{q, A S}=-\beta_{\text {aacbb }}^{q, A S}=\beta_{\text {acaaa }}^{q, A S}=-\beta_{\text {acabb }}^{q, A S}=\beta_{\text {caaaa }}^{q, A S}=-\beta_{\text {caabb }}^{q, A S}=-\beta_{b b c a a}^{q, A S}=\beta_{b b c b b}^{q, A S}=-\beta_{b c b a a}^{q, A S} \\
& =\beta_{b c b b b}^{q, A S}=-\beta_{c b b a a}^{q, A S}=\beta_{c b b b b}^{q, A S}=\beta_{a b c a b}^{q, A S}=\beta_{a b c b a}^{q, A S}=\beta_{a c b a b}^{q, A S}=\beta_{a c b b a}^{q, A S}=\beta_{b a c a b}^{q, A S}=\beta_{b a c b a}^{q, A S} \\
& =\beta_{b c a a b}^{q, A S}=\beta_{b c a b a}^{q, A S}=\beta_{c a b a b}^{q, A S}=\beta_{c a b b a}^{q, A S}=\beta_{c b a a b}^{q, A S}=\beta_{c b a b a}^{q, A S}\left(=R_{A S}^{R} R_{A S, 5}^{H R} \beta_{\text {aaaac }}^{q, A S}\right)
\end{aligned}
$$

From the above discuss, we should note that the two elements $\beta_{i j^{\prime} k^{\prime \prime}}^{q}$ and $\beta_{i{ }^{\prime} j^{\prime \prime} \prime^{\prime}}^{q}$ are unlikeness at some times, although $j$ and $k$ are denote the same frequency. Because the symmetry of the $\beta_{i j^{\prime} k^{\prime \prime}}^{q}$ is decided to the symmetry of the $\alpha_{i j^{\prime}}^{\prime}$ and $\alpha_{k^{\prime \prime}}^{\prime}$, when the symmetry of $\alpha_{i j^{\prime}}^{\prime}$ and $\alpha_{k^{\prime \prime}}^{\prime}$ are unlikeness the symmetry of $\alpha_{i^{\prime} k^{\prime}}^{\prime}$ and $\alpha_{j^{\prime \prime}}^{\prime}$, the two elements $\beta_{i j^{\prime} k^{\prime \prime}}^{q}$ and $\beta_{i^{\prime} k^{\prime \prime} \prime^{\prime}}^{q}$ are unlikeness. For example, toward the $C_{3 \mathrm{~V}}$ symmetry molecule, $\beta_{\text {aacc }}^{q}$ belong to the $A_{1}$ symmetric mode, and $\beta_{\text {acac }}^{q}$ belong to the $E$ symmetric mode.

\section{Conclusion}

Knowledge of the ratios between different polarizability tensor elements of a chemical group in a molecule is crucial for quantitative interpretation and polarization analysis of its coherent anti-stokes raman spectra (CARS) and coherent anti-stokes hyper-raman spectra (CAHRS). The hyperpolarizability derivative model along with experimentally obtained Raman and Hyper-Raman depolarization ratios could be used to obtain such tensor ratios for the $\mathrm{CH} 3, \mathrm{CH} 2$, and $\mathrm{CH}$ groups. Such treatment can be used to quantitatively analyze and simulate the intensity and field polarization dependence in the CARS and CAHRS spectra for the symmetric and asymmetric stretching modes of $\mathrm{CH} 3$ groups, respectively.

\section{Acknowledgement}

This work was supported mainly by the Project Supported by the Scientific Research Fund of the provincial Natural Science Foundation of Hainan(No.114015), the Project Supported by the Scientific Research Fund of the provincial Natural Science Foundation of Hainan(No.614252).

\section{References}

[1] H. F. Wang, W. Gan, R. Lu, Y. Rao, and B. H. Wu, Int. Rev. Phys. Chem. 24, 191 (2005).

[2] J. P. Singh, F. Y. Yueh. Aplied. Optics. 30, 1967 (1991).

[3] Ryoji Inaba, Hiromi Okamoto, Keitaro Yoshihara, Mitsuo Tasumi. J. Phys. Chem. 96, 8385 (1992).

[4] Nancy E. Triggs, Mansour Zahedi, Joseph W. Nibler. J. Chem. Phys. 96, 1822 (1992).

[5] Sukesh Roy, Terrence R. Meyer, Robert P. Lucht, Vincent M. Belovich, Edwin Corporan, James R. GordCombust. Flame.138, 273(2004).

[6] Chaozhi Wan and Carey K. Johnson. J. Chem. Phys. 99, 7602 (1993). 
[7] Andreas Zumbusch, Gary R Holtom, X Sunney Xie. Phys. Rev. Lett. 82, 4142 (1999).

[8] Andreas Volkmer, Ji-Xin Cheng, X Sunney Xie. Phys. Rev. Lett. 87, 023901 (2001).

[9] Ji-Xin Cheng, Sophie Pautot, David A Weitz, X Sunney Xie. Proc. Natl. Acad. Sci.100, 9826 (2003).23

[10] Conor L. Evans, Eric O. Potma, Mehron Puoris'haag, Daniel Cô té, Charles P. Lin, X. Sunney Xie. Proc. Natl. Acad.Sci.102, 16807 (2005).

[11] I. Baltog, M. Baibarac. Phys. Rev. B 72, 245402 (2005).

[12] JACQUES DUCHÊNE, THIBAULT LAMOTTE. Ergonomics. 44, 313 (2001).

[13] Wang H F. Chin. J. Chem. Phys. 17, 362 (2004).

[14] Y. Wang, Z. F. Cui, and H. F. Wang, Chin. J. Chem. Phys., 20, 449 (2007).

[15] Y. R. Shen, The Principles of Nonlinear Optics; John Wiley \& Sons: New York, 1984.

[16] Sang-Ho Lee, Jia Wang, Samuel Krimm, Zhan Chen. J. Phys. Chem. A 110, 7035 (2006).

[17] C. Z. Wan and C. K. Johnson, J. Chem. Phys., 99, 7602 (1993).

[18] M. Yang, J. Kim, Y. Jung, and M. Cho, J. Chem. Phys., 108, 4013 (1998). 